Washington University School of Medicine Digital Commons@Becker

Open Access Publications

2017

\title{
A human antibody against Zika virus crosslinks the E protein to prevent infection
}

\author{
S. Saif Hasan \\ Purdue University \\ Andrew Miller \\ Purdue University \\ Gopal Sapparapu \\ Vanderbilt University Medical Center \\ Estefania Fernandez \\ Washington University School of Medicine in St. Louis \\ Thomas Klose \\ Purdue University
}

See next page for additional authors

Follow this and additional works at: https://digitalcommons.wustl.edu/open_access_pubs

Please let us know how this document benefits you.

\section{Recommended Citation}

Hasan, S. Saif; Miller, Andrew; Sapparapu, Gopal; Fernandez, Estefania; Klose, Thomas; Long, Feng; Fokine, Andrei; Porta, Jason C.; Jiang, Wen; Diamond, Michael S.; Crowe Jr., James E.; Kuhn, Richard J.; and Rossmann, Michael G., "A human antibody against Zika virus crosslinks the E protein to prevent infection." Nature Communications. 8, (2017).

https://digitalcommons.wustl.edu/open_access_pubs/5712

This Open Access Publication is brought to you for free and open access by Digital Commons@Becker. It has been accepted for inclusion in Open Access Publications by an authorized administrator of Digital Commons@Becker. For more information, please contact vanam@wustl.edu. 


\section{Authors}

S. Saif Hasan, Andrew Miller, Gopal Sapparapu, Estefania Fernandez, Thomas Klose, Feng Long, Andrei

Fokine, Jason C. Porta, Wen Jiang, Michael S. Diamond, James E. Crowe Jr., Richard J. Kuhn, and Michael G. Rossmann 
ARTICLE

Received 13 Dec 2016 | Accepted 24 Jan 2017 | Published 16 Mar 2017 DOl: 10.1038/ncomms14722 OPEN

\section{A human antibody against Zika virus crosslinks the E protein to prevent infection}

S. Saif Hasan¹, Andrew Miller ${ }^{1}$, Gopal Sapparapu ${ }^{2,3}$, Estefania Fernandez ${ }^{4}$, Thomas Klose ${ }^{1}$, Feng Long ${ }^{1}$, Andrei Fokine1, Jason C. Porta1, Wen Jiang ${ }^{1,5}$, Michael S. Diamond 4,6,7,8, James E. Crowe Jr. 2,3,9, Richard J. Kuhn ${ }^{1,5}$ \& Michael G. Rossmann ${ }^{1,5}$

The recent Zika virus (ZIKV) epidemic has been linked to unusual and severe clinical manifestations including microcephaly in fetuses of infected pregnant women and Guillian-Barré syndrome in adults. Neutralizing antibodies present a possible therapeutic approach to prevent and control ZIKV infection. Here we present a $6.2 \AA$ resolution three-dimensional cryo-electron microscopy (cryoEM) structure of an infectious ZIKV (strain H/PF/2013, French Polynesia) in complex with the Fab fragment of a highly therapeutic and neutralizing human monoclonal antibody, ZIKV-117. The antibody had been shown to prevent fetal infection and demise in mice. The structure shows that ZIKV-117 Fabs cross-link the monomers within the surface $E$ glycoprotein dimers as well as between neighbouring dimers, thus preventing the reorganization of $E$ protein monomers into fusogenic trimers in the acidic environment of endosomes.

\footnotetext{
${ }^{1}$ Department of Biological Sciences, Purdue University, West Lafayette, Indiana 47907, USA. ${ }^{2}$ Department of Pediatrics, Vanderbilt University Medical Center, Nashville, Tennessee 37232, USA. ${ }^{3}$ The Vanderbilt Vaccine Center, Vanderbilt University Medical Center, Nashville, Tennessee 37232, USA.

${ }^{4}$ Department of Pathology \& Immunology, Washington University School of Medicine, St Louis, Missouri 63110, USA. ${ }^{5}$ Markey Center for Structural Biology and Purdue Institute for Inflammation, Immunology and Infectious Disease, Purdue University, West Lafayette, Indiana 47907, USA. ${ }^{6}$ Department of Medicine, Washington University School of Medicine, St Louis, Missouri 63110, USA. 7 Department of Molecular Microbiology, Washington University School of Medicine, St Louis, Missouri 63110, USA. ${ }^{8}$ Center for Human Immunology and Immunotherapy Programs, Washington University School of Medicine, St Louis, Missouri 63110, USA. ${ }^{9}$ Department of Pathology, Microbiology and Immunology, Vanderbilt University, Nashville, Tennessee 37232 , USA. Correspondence and requests for materials should be addressed to R.J.K. (email: kuhnr@purdue.edu) or to M.G.R. (email: mr@purdue.edu).
} 
Z ika virus (ZIKV) is a member of the Flaviviridae family of positive-stranded RNA viruses, which includes well-known human pathogens such as dengue (DENV), yellow fever (YFV), West Nile (WNV) and Japanese encephalitis (JEV) viruses $^{1}$. ZIKV was first identified in sentinel rhesus monkeys in Uganda in 1947 (ref. 2), in mosquitoes in 1948 (ref. 2) and was first isolated from humans in 1952 (ref. 3). The first major outbreak of the virus was recorded in 2007 in Micronesia ${ }^{4}$ and then in 2013-2014 in Oceania $^{5}$. The latest outbreak, which started in Brazil in 2014-2015 (ref. 6), has spread to other countries in South America, North America and the Caribbean 7 .

Flaviviruses contain an RNA genome $(\sim 11 \mathrm{~kb})$ that is enclosed within the viral capsid. The nucleocapsid complex is enclosed within an icosahedral shell formed by 180 copies of each of the envelope (' $\mathrm{E}$ ', $\sim 500$ residues) and membrane ('M', $\sim 75$ residues) glycoproteins. The $\mathrm{M}$ protein is a product of cleavage of the precursor M ('prM', 165 residues) protein. Cryo-electron microscopy (cryoEM) structures of mature ZIKV (refs 8,9) showed a typical 'herringbone' arrangement of the E proteins on the viral surface, which is a characteristic of mature flaviviruses ${ }^{10}$. Following cellular entry, flaviviruses assemble first as 'spiky', non-infectious immature particles with 60 trimeric spikes of E:prM heterodimers ${ }^{11,12}$. The immature particles undergo maturation into smooth infectious particles, which involves the cleavage of the prM protein into $\mathrm{pr}$ and $\mathrm{M}$. The $\mathrm{E}$ and $M$ glycoproteins are reorganized into 90 dimers ${ }^{11,12}$. Upon release into the extracellular environment, the pr peptide dissociates from the flavivirus surface to yield smooth, mature, infectious particles $^{11,13}$.

ZIKV infections were previously associated with only a rash and mild flu-like symptoms until the latest epidemic, during which a link was established between ZIKV infection and paralytic Guillian-Barré syndrome in adults ${ }^{14}$, and fetal abnormalities in pregnant women, including microcephaly ${ }^{15,16}$. Four cases of fetal deformities have been reported in December 2016 in New York City ${ }^{17}$. In contrast to other flaviviruses that are spread mainly by arthropod vectors, recent evidence suggests that ZIKV can be transmitted sexually and vertically in addition to transmission by mosquitoes ${ }^{18,19}$. Given the severity of the symptoms caused by ZIKV infection in humans, it is crucial to understand the immune response elicited by ZIKV infection to develop neutralizing anti-ZIKV therapies. A recent study identified a human monoclonal antibody (mAb), ZIKV-117, that was demonstrated to possess therapeutic potential, with no cross-reactivity to other flaviviruses ${ }^{20}$. This is significant as ZIKV antibodies that cross-react with DENV have been shown to promote DENV infection by antibody dependent enhancement ${ }^{21}$. The mAb ZIKV-117 neutralizes ZIKV strains that belong to African, Asian and American lineages and is able to reduce fetal infection and death in mice. However, the mechanisms of neutralization of ZIKV infection by mAb ZIKV-117 and the structural basis for broad-range neutralization of ZIKV strains, while showing no cross-reactivity with other flaviviruses, had remained unknown.

Here we present a cryoEM map of ZIKV strain H/PF/2013 in complex with ZIKV-117 Fab at a resolution of $6.2 \AA$. The map showed that the mechanism of virus neutralization was by binding of Fab fragments that cross-linked the $\mathrm{E}$ monomers within dimers and also cross-linked neighbouring dimers in the viral glycoprotein shell. However, the binding of any one of the Fab molecules excluded binding of a Fab to any of the other chemically equivalent sites in the icosahedral asymmetric unit. Thus, saturation is achieved by binding of only 60 Fabs to the 180 chemically equivalent sites on the virus, greatly reducing the concentration of the antibody needed for neutralization.

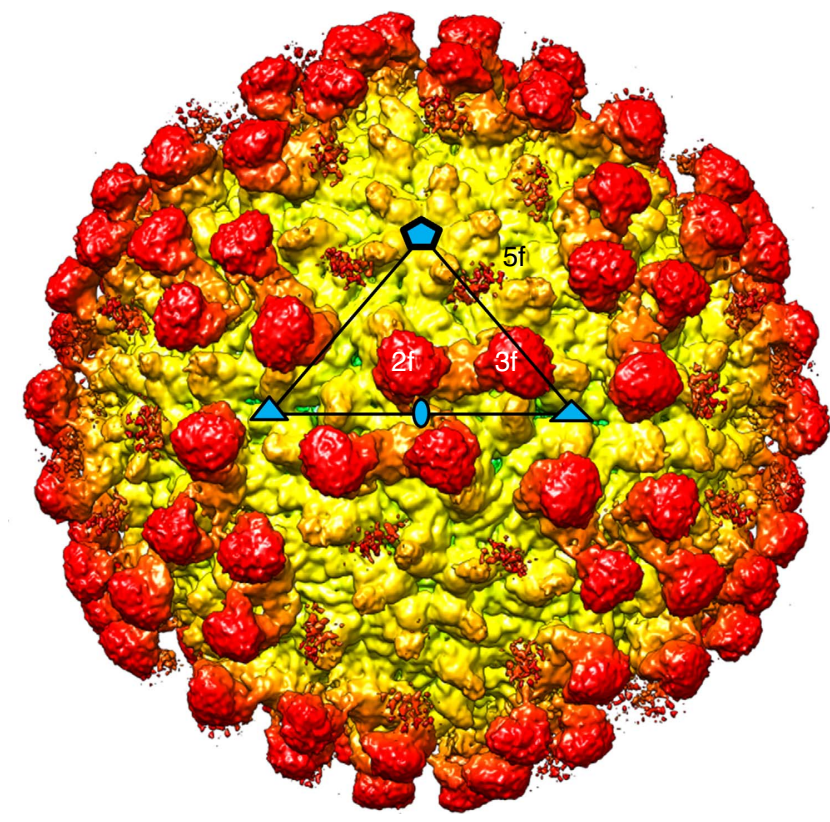

Figure 1 | CryoEM map of ZIKV/ZIKV-117. The ZIKV-Fab surface is shown in radial colouring, from yellow $(\sim 210 \AA)$ to red $(\sim 290 \AA)$. The bound Fab (red 'knobs') extends from the surface of ZIKV, which is shown in yellow. The Fab sites close to the two-fold (' $2 f$ '), three-fold (' $3 f$ ') and

five-fold (' $5 f^{\prime}$ ) axes are labelled. The icosahedral 2 (oval), 3 (triangle) and 5 (pentagon) fold axes are marked in blue shapes with black outlines, and a representative asymmetric unit is shown as a black triangle.

\section{Results}

The cryoEM structure of ZIKV complexed with ZIKV-117 Fab. Mature ZIKV was purified from Vero cells over-expressing the enzyme furin ${ }^{8}$. MAb ZIKV-117 was isolated from a B cell in a peripheral blood sample, obtained from an otherwise healthy human subject with previous history of symptomatic ZIKV infection $^{20}$. The ZIKV particles were incubated with Fab fragments of ZIKV-117, flash-frozen on lacey carbon EM grids and imaged using a Gatan K2 direct electron detector attached to a Titan-Krios microscope. After automated selection of virus-Fab particles, a data set containing 8,153 virus-Fab complexes was split into two halves to perform two independent 3D-reconstructions for a 'gold-standard' evaluation of the resolution ${ }^{22}$. The previously published ZIKV cryoEM map ${ }^{8}$ was low-pass filtered to $50 \AA$ resolution, and used as the initial model for single particle reconstruction by the program jspr (ref. 23). The final map had a resolution of $6.2 \AA$ corresponding to a Fourier Shell Correlation coefficient of 0.143 (ref. 24) (Supplementary Fig. 1). The cryoEM map (Fig. 1) was interpreted by fitting of $\mathrm{E}$ protein and Fab coordinates with the programs Chimera ${ }^{25}$, Coot $^{26}$ and EMfit ${ }^{27}$ following the original procedure first used to determine the structure of DENV ${ }^{10}$. The icosahedral asymmetric unit of the ZIKV structure contains three copies of the $\mathrm{E}$ protein, whose ectodomain is organized into three domains, DI, DII and DIII (ref. 28) (Supplementary Fig. 2a,b). The structure of a ZIKV E dimer (Protein Data Bank, PDB ID 5IRE, residues 1-396) (ref. 8) was fitted into the cryoEM map by superposing the dimer two-fold axis onto an icosahedral two-fold axis ('i2 axis') (Fig. 2, monomers $A$ and $A^{\prime}$ ) using the Chimera and EMfit programs. This dimer will be referred to as the icosahedral E dimer ('i2 dimer'). A second E dimer (Fig. 2, monomers $\mathrm{C}$ and $\mathrm{E}$ ) was fitted at a general position, where the cryoEM map appears to have a quasi two-fold axis ('q2 axis', Fig. 2, yellow oval). This dimer will be referred to as the general 


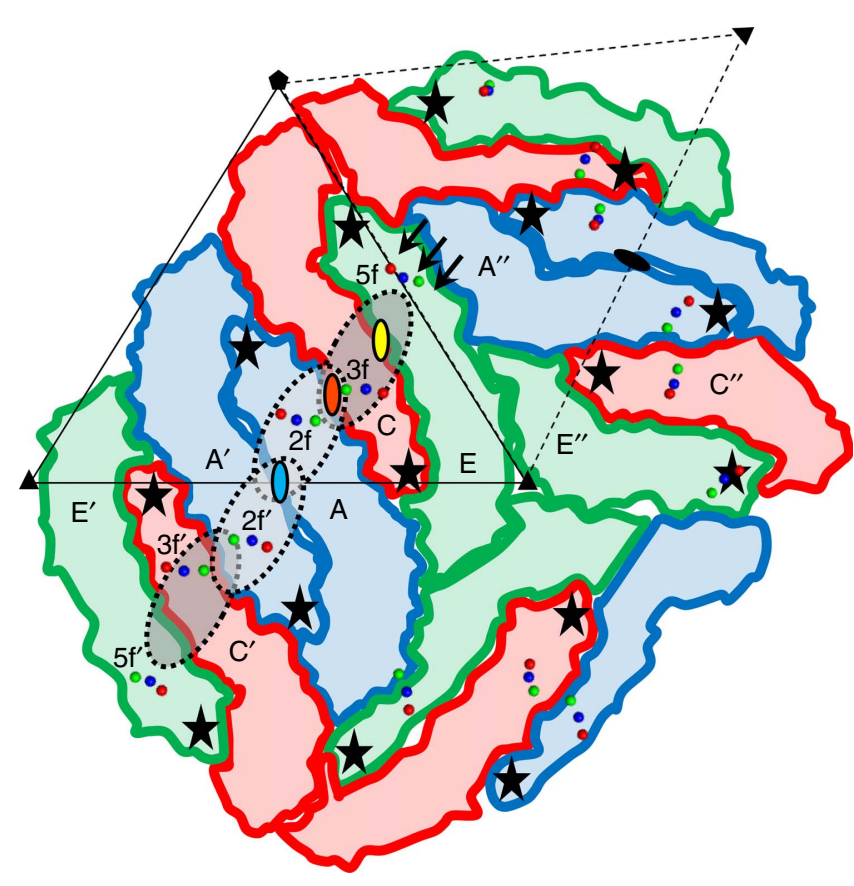

Figure 2 | Fab binding sites on ZIKV surface. The $E$ proteins are represented in three colours-blue (chains $A, A^{\prime}$ and $A^{\prime \prime}$ ), red (chains $C, C^{\prime}$ and $C^{\prime \prime}$ ) and green (chains $E, E^{\prime}$ and $E^{\prime \prime}$ ). Chains $A$ and $A^{\prime}$ are arranged in a dimer around the icosahedral two-fold axis (blue oval with black outline), while chains $C$ and $E$ are arranged in a general dimer around a quasi two-fold axis (yellow oval with black outline). The icosahedral and general dimers are related by an axis of quasi two-fold symmetry (orange oval with black outline). Residues Asp67 (red), Gln89 (green) and Lys118 (blue), which were mapped to the Fab binding site by mutagenesis, are shown as spheres. The footprints of the Fab bound at the $2 f$ and $3 f$ sites are represented as light and dark grey ovals with broken outlines. The black stars mark the position of the fusion peptides. The steric hindrance on the $5 f$ site due to the orientation of DIII of chain $A^{\prime \prime}$ is highlighted by black arrows. For reference, two asymmetric units are shown as triangles in solid and broken dashed lines. The icosahedral five- and three-fold axes are marked with a pentagon and two triangles receptively.

E dimer ('q2 dimer'). As the asymmetric unit contains three copies of the E protein (Fig. 2, monomers A, C and E), it should be possible for three Fabs to be bound within the asymmetric unit. However, only two prominent Fab-bound positions were observed, one (' $2 \mathrm{f}$ ) closest to an $\mathrm{i} 2$ axis and the other (' $3 \mathrm{f}$ ) closest to an icosahedral three-fold axis ('i3 axis') (Figs 1 and 2). The additional density at the third chemically equivalent position (' $5 f$ ) closest to an icosahedral five-fold axis ('i5 axis') was weak (Fig. 1). The coordinates of a ZIKV-specific Fab (ZV-67, PDB ID 5KVG) (ref. 29) were split into the constant and variable domains of the heavy and light chains. These domains were fitted individually into the Fab densities at the $2 \mathrm{f}$ and $3 \mathrm{f}$ sites using Chimera and Coot. As the density is incomplete at the $5 f$ site, Fab coordinates were placed by hand. The average heights of electron density of the E proteins and the Fabs were compared using the program EMfit.

Interference between overlapping ZIKV-117 Fab binding sites. Within an icosahedral asymmetric unit of ZIKV, the two E protein monomers of the general dimer (Fig. 2, monomers $\mathrm{C}$ and $\mathrm{E}$ ) are related by q2 symmetry (Fig. 2, yellow oval). The icosahedral $\mathrm{E}$ dimer (Fig. 2, monomers $\mathrm{A}$ and $\mathrm{A}^{\prime}$ ) is similar in structure to the general $\mathrm{E}$ dimer (Fig. 2, monomers $\mathrm{C}$ and $\mathrm{E}$ ). As a result, the icosahedral i2 dimer and the general q2 dimer are related by a q2 axis (Fig. 2, orange oval). Three residues of the ZIKV E protein, Asp67, Gln89 and Lys118 ('Asp-Gln-Lys triad'), previously determined to be in the ZIKV-117 epitope by alanine mapping ${ }^{20}$, are located closest to the $\mathrm{q} 2$ axis (Fig. 2, orange oval) that relates the i2 dimer with the general $\mathrm{q} 2$ dimer. The similar positions of these three residues in the footprint of the Fabs at both the $2 \mathrm{f}$ and $3 \mathrm{f}$ sites are satisfactorily consistent with the EM results presented here and also demonstrate the chemical equivalence of these sites.

Binding of Fabs at the $5 \mathrm{f}$ sites around each five-fold axis is not limited by overlap between Fabs bound to neighbouring $5 \mathrm{f}$ sites because these sites are spatially well separated (Supplementary Fig. 3). However, the low occupancy of Fabs at the supposedly chemically equivalent $5 \mathrm{f}$ sites is readily understandable because of the different environments of the $5 \mathrm{f}$ sites to the $3 \mathrm{f}$ and $2 \mathrm{f}$ sites. The 'road map' (Fig. 3) shows that the 5 f site is bounded at one side by an E monomer that extends radially outwards further than the $5 \mathrm{f}$ site. Therefore, the E monomer not only changes a part of the $5 \mathrm{f}$ site to be different to the $3 \mathrm{f}$ and $2 \mathrm{f}$ sites, but also sterically hinders the binding of Fabs to the $5 f$ sites. The residual small occupancy of the $5 \mathrm{f}$ sites (Fig. 1) is probably due to the inclusion of particles in the cryoEM reconstruction that were able to bind $\mathrm{Fab}$ at the $5 \mathrm{f}$ sites after inducing small local conformational changes.

The footprints of Fabs when bound to the $3 \mathrm{f}$ and $2 \mathrm{f}$ sites overlap (Figs 2 and 3). This implies that binding of Fabs at these sites within an icosahedral asymmetric unit is mutually exclusive. Only one Fab can be bound within an asymmetric unit, either at the $2 \mathrm{f}$ or the $3 \mathrm{f}$ site. Furthermore, the footprint of the $2 f$ site overlaps the footprint of the two-fold related $2 f$ site (Figs 2 and 3). Thus, similarly only one of the $2 \mathrm{f}$ or $2 \mathrm{f}^{\prime}$ sites can be occupied at a time. Lastly the binding of Fab to the $2 \mathrm{f}^{\prime}$ and $3 \mathrm{f}^{\prime}$ sites is also mutually exclusive. Assuming that each binding site is chemically identical and therefore equally likely to bind a Fab molecule, there are three different possible arrangements of Fabs that can bind to the overlapping $3 \mathrm{f}, 2 \mathrm{f}, 2 \mathrm{f}^{\prime}$ and $3 \mathrm{f}^{\prime}$ sites (Supplementary Fig. 4). Inspection of Supplementary Fig. 4 shows that on average, the $3 \mathrm{f}$ and $3 \mathrm{f}^{\prime}$ sites will be occupied two times out of every three randomly chosen particles, whereas the $2 \mathrm{f}$ and $2 \mathrm{f}^{\prime}$ sites will be occupied only once out of three randomly chosen particles. Thus, on average, the $3 \mathrm{f}$ site should have $67 \%$ occupancy whereas the $2 \mathrm{f}$ site should have $33 \%$ occupancy.

The Fab occupancy at the $2 \mathrm{f}$ and $3 \mathrm{f}$ sites relative to the ZIKV E protein was determined by the program EMfit, which calculates the average electron density at the atomic centres of the fitted coordinates. The average heights of density of the Fabs bound at the $2 \mathrm{f}$ and $3 \mathrm{f}$ sites were 35 and $58 \%$ respectively of the E protein density (100\% occupancy, Supplementary Table 1), which correspond to 21 and 35 Fabs at the $2 \mathrm{f}$ and $3 \mathrm{f}$ sites. The missing $7 \%$ of Fabs is either because the average of all the particles used in the EM reconstruction had less than 60 Fabs per particle or is simply experimental error. Given the experimental conditions, the observed occupancies are in excellent agreement with the predicted 33 and 67\% (Supplementary Fig. 4).

\section{Discussion}

The efficacy of neutralization of ZIKV infection by ZIKV-117 is in part due to the requirement of only 60 bound Fab molecules instead of the anticipated stoichiometric 180 bound Fabs to effectively cross-link the glycoprotein shell. In addition, the tight binding of the $\mathrm{Fab}$ to the $\mathrm{E}$ protein is also a significant contributing factor. The footprints of the Fabs bound at the $2 \mathrm{f}$ and $3 \mathrm{f}$ sites consist primarily of polar residues of the E protein (Fig. 3), which can participate in the formation of salt bridges and 


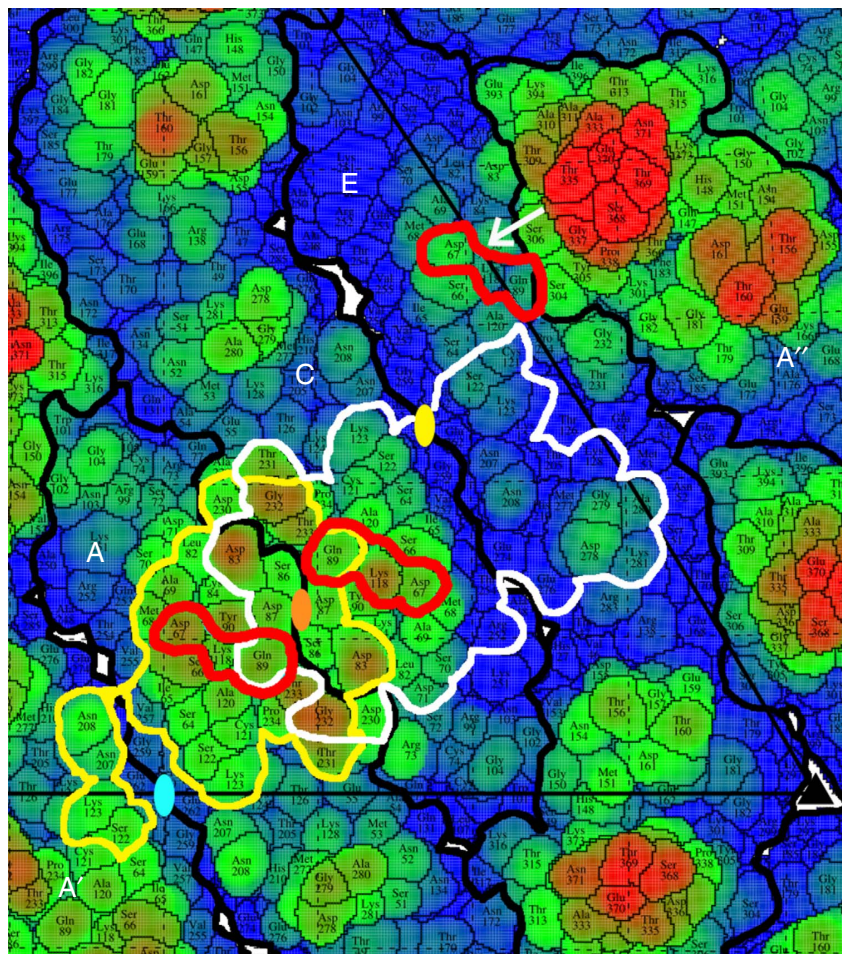

Figure 3 | Footprints of ZIKV-117 Fabs on the E protein. The figure shows a roadmap of the ZIKV E protein. The E protein residues are coloured according to their distance from the virus centre, with the residues closer to the centre coloured blue $(218 \AA)$ and those farthest from the centre coloured red $(235 \AA)$. The colours of residues transition from blue to red through green. The position of the icosahedral two-fold axis is marked with a blue oval. The quasi two-fold axes are marked as orange or yellow ovals. The outlines of the individual $\mathrm{E}$ protein chains are marked with thick black lines, and the chains are labelled as per the convention described in Fig. 2. The residues Asp67, GIn89 and Lys118 within the ZIKV-117 epitope are highlighted with red lines. The ZIKV-117 footprints formed by E protein residues that lie within $8 \AA$ of the bound Fab at the $2 f$ (yellow outline) and $3 f$ (white outline) sites are highlighted across the quasi two-fold axis

(orange oval). The footprints extend across multiple E proteins, and there is significant overlap between the footprints. At the $5 f$ site, DIII of chain $A^{\prime \prime}$ (highlighted with a white arrow) poses a steric barrier to the binding of Fab.

hydrogen bonds with the ZIKV-117 Fab to form strong, stable virus-Fab interactions. In addition, the Fab footprints show that ZIKV-117 cross-links E monomers within a dimer, and across neighbouring dimers (Fig. 3). At the $2 \mathrm{f}$ site, the Fab cross-links monomers $\mathrm{A}$ and $\mathrm{A}^{\prime}$ of the $\mathrm{i} 2$ dimer and monomer $\mathrm{C}$ of the general q2 dimer (Figs 2 and 3). In contrast, the Fab bound at the 3 site cross-links monomers $\mathrm{C}$ and $\mathrm{E}$ of the general q2 dimer and monomer A of the i2 dimer (Figs 2 and 3). Therefore, ZIKV-117 causes extensive intra-dimer as well as inter-dimer cross-linking of the ZIKV E protein.

Antibodies that cross-link E proteins and neutralize infection have been reported for $\mathrm{ZIKV}^{30}, \mathrm{DENV}^{31-35}$ and $\mathrm{WNV}^{36}$. These antibodies cross-link DII with either DI or DIII, or with both DI and DIII. The ZIKV-specific antibody binds to DIII at 120 of 180 sites, away from the $\mathrm{E}$ protein dimer axis ${ }^{30}$. A structure of a DENV antibody bound to ZIKV shows that the antibody binds at 180 sites on the virus particle to cross-link DI, DII and DIII to prevent low $\mathrm{pH}$ triggered conformational changes of the virus $^{37}$. The epitopes of the cross-linking ZIKV, DENV and $\mathrm{WNV}$ antibodies are not close to the $\mathrm{i} 2$ or $\mathrm{q} 2$ axes. Therefore, in contrast to ZIKV-117, the binding of Fab to one site does not exclude binding of $\mathrm{Fab}$ to another site. This implies that antibodies that bind to sites on DII are likely to require a lower concentration to saturate and neutralize the virus.

The flavivirus $\mathrm{E}$ dimer is converted into a trimer upon viral entry into host cells under acidic conditions in endosomes ${ }^{38-40}$. These trimers expose the fusion peptides which insert themselves into the endosomal membrane to form a channel across the endosomal membrane for the transfer of the viral RNA genome into the host's cytosol ${ }^{41}$. Binding of the ZIKV-117 Fab at the $2 \mathrm{f}$ and $3 \mathrm{f}$ positions will inhibit the low-pH triggered rearrangement of the E protein dimer into a fusogenic trimer inside the host cell. This is supported by a pre-/post-neutralization assay of ZIKV infection, which shows that the ZIKV-117 is able to significantly inhibit infection at a post-attachment, intra-cellular step (Supplementary Fig. 5).

At acidic $\mathrm{pH}$ mature flaviviruses undergo a major conformational change in which the E glycoproteins re-arrange themselves to form 60 trimeric spikes ${ }^{42}$ with the fusion peptide in DII of each monomer exposed at their distal extremity. This large re-arrangement is likely to leave the virus lacking homogeneity accounting for the decline in obtaining high resolution cryoEM structures ${ }^{37}$. Inspection of Fig. 2 suggests various ways in which the monomers might re-arrange themselves to extend radially with their fusion peptides at the far end of the spikes away from the virus centre. For instance, monomers A, C and E (Fig. 2) are close together to form a spike, and monomers $\mathrm{A}^{\prime}, \mathrm{C}^{\prime}$ and $\mathrm{E}^{\prime}$ would then be able to form a two-fold related spike. This would allow the formation of one spike per icosahedral asymmetric unit. However if monomers $\mathrm{A}^{\prime}, \mathrm{A}$ and $\mathrm{C}$ were to form a spike, they would leave monomers $\mathrm{E}, \mathrm{C}^{\prime}, \mathrm{E}^{\prime}$ without partners. This would allow the formation of one spike for two icosahedral asymmetric units. But acidification of DENV results in the formation of 60 spikes per particle, which is one spike per asymmetric unit ${ }^{41}$. Therefore, the first option mentioned here is a more likely association of monomers that make a fusogenic virus. As the overlap of ZIKV-117 Fab binding sites especially emphasizes the cross linking of monomers A, C and E, it is probable that the antibody will inhibit the formation of fusogenic spikes. This also shows that the binding of $60 \mathrm{Fabs}$ is enough to inhibit $90 \mathrm{E}$ dimers from making a spikey fusogenic structure.

Since the beginning of the 2013 ZIKV outbreak, emphasis has been placed on the study of cross-reacting anti-DENV antibodies for neutralizing ZIKV infection ${ }^{21,37,43-46}$. In vivo studies have shown that anti-ZIKV antibodies that cross-react with DENV may promote infection by antibody-dependent enhancement ${ }^{21}$. The ZIKV-117 mAb does not bind any of the four DENV serotypes or $\mathrm{WNV}^{20}$. An alignment of flavivirus sequences shows that the E protein residues that form the ZIKV-117 footprint are poorly conserved across flaviviruses (Supplementary Fig. 6a). In contrast, the footprint of ZIKV-117 is largely conserved among ZIKV strains (Supplementary Fig. 6b). Consistent with recent functional data showing potent neutralization of five different African, Asian and American strains of ZIKV, the ZIKV-117 $\mathrm{mAb}$ binds to a conserved ZIKV epitope and should therefore provide broad-range protection against virtually all strains of ZIKV.

In summary, considering that the surface of ZIKV is made of 60 copies of three environmentally different $\mathrm{E}$ monomers, it would be expected that 180 copies of the Fab would be bound to the ZIKV virion. Our study shows that contrary to expectation, Fab binding at the $5 f$ site is extremely weak despite no obvious clashes between symmetry related Fabs around the five-fold axes. We show that the epitope at the $5 f$ site has limited accessibility due to steric hindrance from a neighbouring $\mathrm{E}$ monomer. Furthermore, our study shows that the overlap between Fab 
binding sites limits the occupancies of the $3 \mathrm{f}$ and $2 \mathrm{f}$ sites in a predictable manner, implying that neutralization of the virus can be achieved with a lower occupancy of antibodies compared to what would be the case were the binding sites well separated. The structure of the Fab-virus complex suggests that the antibody functions by preventing the rearrangement of $\mathrm{E}$ proteins necessary for low-pH mediated fusion.

\section{Methods}

Isolation of ZIKV-117 Fab fragments. MAb ZIKV-117 is a naturally occurring fully human $\mathrm{mAb}$ isolated from a $\mathrm{B}$ cell in a peripheral blood sample obtained from an otherwise healthy subject with previous history of symptomatic ZIKV infection. The isolation and determination of the variable region sequences of the heavy and light chains were described previously ${ }^{20}$. RT-PCR was performed with primers having overlapping regions with the leader and constant domains ${ }^{47}$, and the amplicons were cloned directly into immunoglobulin expression vector cassettes ${ }^{48}$ using a Gibson assembly kit (New England Biolabs). Plasmids encoding the heavy or light chains were transfected transiently into Expi293 cells (Invitrogen), and the secreted Fab protein was harvested from culture supernatant. Recombinant Fab protein was concentrated and purified by affinity chromatography using the CaptureSelect IgG-CH1 matrix (Thermo Scientific) and further concentrated using 10 MWCO concentrators (Millipore).

CryoEM and 3D reconstruction. The ZIKV particles were incubated with the ZIKV-117 Fab at $4{ }^{\circ} \mathrm{C}$ for $2-3 \mathrm{~h}$, and then flash-frozen $(2.5 \mu \mathrm{l})$ on lacey carbon EM grids by plunging in liquid ethane using a Gatan Cp3 plunger. Micrographs were recorded on a Gatan K2 direct electron detector, attached to a Titan-Krios microscope at a magnification of $\times 18,000$ in the direct counting mode with a pixel size of $1.62 \AA$. The total electron dose per image was $\sim 30 \mathrm{e} \AA^{-2}$. The automated software Leginon ${ }^{49}$ was used to collect 1,141 micrographs, of which 406 micrographs were rejected due to poor ice thickness, drift and errors in defocus. A total of 38,835 particles of the Fab-virus complex were selected in Appion ${ }^{50}$ from the remaining 735 micrographs, within a defocus range of -2.5 to $-5.0 \mu \mathrm{m}$. The 38,853 particle set was then subjected to two rounds of non-referenced 2Dclassification in RELION ${ }^{51}$ to generate a subset of 8,153 homogeneous Fab-virus particles, which was split into two half data sets to perform two independent $3 \mathrm{D}$-reconstructions for a 'gold-standard' evaluation of the resolution ${ }^{22}$. The previously published ZIKV cryoEM map ${ }^{8}$ was low-pass filtered to $50 \AA$ resolution, and used as the initial model for single particle reconstruction by the program $j s p r$ (ref. 23). During the initial stages of the reconstruction, the centre position and orientation of each particle were refined using projection matching, which was followed by the refinement of high-order parameters, that is, anisotropic magnification, astigmatism, defocus and beam tilt ${ }^{52,53}$. A resolution of $6.2 \AA$ was assigned corresponding to a Fourier Shell Correlation coefficient of 0.143 (Supplementary Fig. 1). The fitting procedure for the E protein and Fab has been described in the main text.

Pre- and post-attachment neutralization assays. For the pre-attachment assay, serial dilutions of recombinant ZIKV-117 were pre-incubated with $10^{2} \mathrm{FFU}$ of ZIKV Brazil Paraiba 2015 for $1 \mathrm{~h}$ at $4{ }^{\circ} \mathrm{C}$. The mAb-virus complexes then were added to Vero cell monolayers in 96-well plates for $1 \mathrm{~h}$ at $4{ }^{\circ} \mathrm{C}$. For the postattachment assay, $10^{2}$ FFU of ZIKV Brazil Paraiba 2015 was added to a Vero cell monolayer in 96-well plates for $1 \mathrm{~h}$ at $4^{\circ} \mathrm{C}$. Unbound virus was removed followed by serial washes with Dulbecco's modified Eagle medium (DMEM), and then dilutions of recombinant ZIKV-117 were added for $1 \mathrm{~h}$ at $4{ }^{\circ} \mathrm{C}$. Subsequently, all cells were washed three times with DMEM, incubated in DMEM supplemented with $5 \% \mathrm{FBS}$ at for $1 \mathrm{~h}$ at $37^{\circ} \mathrm{C}$, and then overlaid with $1 \%(\mathrm{w} / \mathrm{v})$ methylcellulose in $\alpha$-MEM supplemented with $4 \%$ heat-inactivated FBS. Plates were fixed $40 \mathrm{~h}$ later with $1 \%$ paraformaldehyde in PBS for $1 \mathrm{~h}$ at room temperature. The plates were incubated sequentially with $500 \mathrm{ng} \mathrm{ml}^{-1}$ mouse anti-ZIKV (ZV-16, E. Fernandez and M. Diamond, unpublished) and HRP-conjugated goat anti-mouse IgG in PBS supplemented with $0.1 \%(\mathrm{w} / \mathrm{v})$ saponin (Sigma) and $0.1 \%$ BSA. ZIKV-infected cell foci were visualized using TrueBlue peroxidase substrate (KPL) and quantitated on an ImmunoSpot 5.0.37 macroanalyzer (Cellular Technologies).

Sequence alignments. The sequences of flavivirus ZIKV E protein were obtained from GenBank. Alignment of E protein sequences was performed using the program Clustal-Omega ${ }^{54}$ and figures were prepared using the program ESpript ${ }^{55}$

Data availability. The cryoEM map and fitted coordinates of the ZIKV/ZIKV-117 complex have been deposited in the Electron Microscopy Data Bank and the Protein Data Bank under the accession codes EMD-8548 and 5UHY respectively. The data that support the findings of this study are available from the corresponding author on request. Requests for antibody material should be addressed to james.crowe@vanderbilt.edu.

\section{References}

1. Kuno, G., Chang, G. J., Tsuchiya, K. R., Karabatsos, N. \& Cropp, C. B. Phylogeny of the genus Flavivirus. J. Virol. 72, 73-83 (1998).

2. Dick, G. W., Kitchen, S. F. \& Haddow, A. J. Zika virus. I. Isolations and serological specificity. Trans. R. Soc. Trop. Med. Hyg. 46, 509-520 (1952).

3. MacNamara, F. N. Zika virus: a report on three cases of human infection during an epidemic of jaundice in Nigeria. Trans. R. Soc. Trop. Med. Hyg. 48, 139-145 (1954).

4. Duffy, M. R. et al. Zika virus outbreak on Yap Island, Federated States of Micronesia. N. Engl. J. Med. 360, 2536-2543 (2009).

5. Cao-Lormeau, V. M. et al. Zika virus, French polynesia, South pacific, 2013. Emerg. Infect. Dis. 20, 1085-1086 (2014).

6. Zanluca, C. et al. First report of autochthonous transmission of Zika virus in Brazil. Mem. Inst. Oswaldo Cruz 110, 569-572 (2015).

7. CDC. All countries \& territories with active Zika virus transmission https:// www.cdc.gov/zika/geo/active-countries.html (2016).

8. Sirohi, D. et al. The $3.8 \AA$ resolution cryo-EM structure of Zika virus. Science 352, 467-470 (2016).

9. Kostyuchenko, V. A. et al. Structure of the thermally stable Zika virus. Nature 533, 425-428 (2016).

10. Kuhn, R. J. et al. Structure of dengue virus: implications for flavivirus organization, maturation, and fusion. Cell 108, 717-725 (2002).

11. Perera, R. \& Kuhn, R. J. Structural proteomics of dengue virus. Curr. Opin. Microbiol. 11, 369-377 (2008).

12. Zhang, Y. et al. Structures of immature flavivirus particles. $E M B O$ J. 22, 2604-2613 (2003).

13. $\mathrm{Yu}, \mathrm{I}$. M. et al. Structure of the immature dengue virus at low $\mathrm{pH}$ primes proteolytic maturation. Science 319, 1834-1837 (2008).

14. Cao-Lormeau, V. M. et al. Guillain-Barre Syndrome outbreak associated with Zika virus infection in French Polynesia: a case-control study. Lancet 387, 1531-1539 (2016).

15. Mlakar, J. et al. Zika virus associated with microcephaly. N. Engl. J. Med. 374, 951-958 (2016).

16. Martines, R. B. et al. Notes from the field: evidence of Zika virus infection in brain and placental tissues from two congenitally infected newborns and two fetal losses-Brazil, 2015. MMWR Morb. Mortal. Wkly. Rep. 65, 159-160 (2016).

17. NYC Health. Health department reports four more babies born with congenital Zika virus syndrome in NYC http://www1.nyc.gov/site/doh/about/press/ pr2016/pr101-16.page (2016)

18. Foy, B. D. et al. Probable non-vector-borne transmission of Zika virus, Colorado, USA. Emerg. Infect. Dis. 17, 880-882 (2011).

19. Musso, D. et al. Potential sexual transmission of Zika virus. Emerg. Infect. Dis 21, 359-361 (2015)

20. Sapparapu, G. et al. Neutralizing human monoclonal antibodies prevent Zika virus replication and fetal disease in pregnancy. Nature 540, 443-447 (2016).

21. Stettler, K. et al. Specificity, cross-reactivity, and function of antibodies elicited by Zika virus infection. Science 353, 823-826 (2016).

22. Scheres, S. H. \& Chen, S. Prevention of overfitting in cryo-EM structure determination. Nat. Methods 9, 853-854 (2012).

23. Guo, F. \& Jiang, W. Single particle cryo-electron microscopy and 3-D reconstruction of viruses. Methods Mol. Biol. 1117, 401-443 (2014)

24. Henderson, R. et al. Tilt-pair analysis of images from a range of different specimens in single-particle electron cryomicroscopy. J. Mol. Biol. 413, 1028-1046 (2011).

25. Pettersen, E. F. et al. UCSF Chimera-a visualization system for exploratory research and analysis. J. Comput. Chem. 25, 1605-1612 (2004).

26. Emsley, P. \& Cowtan, K. Coot: model-building tools for molecular graphics. Acta Crystallogr. D Biol. Crystallogr. 60, 2126-2132 (2004).

27. Rossmann, M. G., Bernal, R. \& Pletnev, S. V. Combining electron microscopic with x-ray crystallographic structures. J. Struct. Biol. 136, 190-200 (2001).

28. Rey, F. A., Heinz, F. X., Mandl, C., Kunz, C. \& Harrison, S. C. The envelope glycoprotein from tick-borne encephalitis virus at $2 \AA$ resolution. Nature 375, 291-298 (1995).

29. Zhao, H. et al. Structural basis of Zika virus-specific antibody protection. Cell 166, 1016-1027 (2016)

30. Wang et al. Molecular determinants of human neutralizing antibodies isolated from a patient infected with Zika virus. Sci. Transl. Med. 369, 369ra179 (2016).

31. Rouvinski, A. et al. Recognition determinants of broadly neutralizing human antibodies against dengue viruses. Nature 520, 109-113 (2015).

32. Dejnirattisai, W. et al. A new class of highly potent, broadly neutralizing antibodies isolated from viremic patients infected with dengue virus. Nat. Immunol. 16, 170-177 (2015). 
33. Fibriansah, G. et al. A highly potent human antibody neutralizes dengue virus serotype 3 by binding across three surface proteins. Nat. Commun. 6, 6341 (2015).

34. Fibriansah, G. et al. Cryo-EM structure of an antibody that neutralizes dengue virus type 2 by locking E protein dimers. Science 349, 88-91 (2015).

35. Teoh, E. P. et al. The structural basis for serotype-specific neutralization of dengue virus by a human antibody. Sci. Transl. Med. 4, 139 ra83 (2012).

36. Kaufmann, B. et al. Neutralization of West Nile virus by cross-linking of its surface proteins with Fab fragments of the human monoclonal antibody CR4354. Proc. Natl Acad. Sci. USA 107, 18950-18955 (2010).

37. Zhang, S. et al. Neutralization mechanism of a highly potent antibody against Zika virus. Nat. Commun. 7, 13679 (2016).

38. Bressanelli, S. et al. Structure of a flavivirus envelope glycoprotein in its low-pH-induced membrane fusion conformation. EMBO J. 23, 728-738 (2004).

39. Modis, Y., Ogata, S., Clements, D. \& Harrison, S. C. Structure of the dengue virus envelope protein after membrane fusion. Nature 427, 313-319 (2004).

40. Zhang, Y. et al. Conformational changes of the flavivirus E glycoprotein. Structure 12, 1607-1618 (2004).

41. Lindenbach, B. D. \& Rice, C. M. Molecular biology of flaviviruses. Adv. Virus Res. 59, 23-61 (2003).

42. Zhang, X. et al. Structure of acidic $\mathrm{pH}$ dengue virus showing the fusogenic glycoprotein trimers. J. Virol. 89, 743-750 (2015).

43. Swanstrom, J. A. et al. Dengue virus envelope dimer epitope monoclonal antibodies isolated from dengue patients are protective against Zika virus. MBio 7, e01123-16 (2016).

44. Dejnirattisai, W. et al. Dengue virus sero-cross-reactivity drives antibodydependent enhancement of infection with Zika virus. Nat. Immunol. 17, 1102-1108 (2016).

45. Barba-Spaeth, G. et al. Structural basis of potent Zika-dengue virus antibody cross-neutralization. Nature 536, 48-53 (2016).

46. Priyamvada, L. et al. Human antibody responses after dengue virus infection are highly cross-reactive to Zika virus. Proc. Natl Acad. Sci. USA 113, $7852-7857$ (2016).

47. Thornburg, N. J. et al. H7N9 influenza virus neutralizing antibodies that possess few somatic mutations. J. Clin. Invest. 126, 1482-1494 (2016).

48. Sapparapu, G. et al. Frequent use of the IgA isotype in human B cells encoding potent norovirus-specific monoclonal antibodies that block HBGA binding. PLoS Pathog. 12, e1005719 (2016).

49. Carragher, B. et al. Leginon: an automated system for acquisition of images from vitreous ice specimens. J. Struct. Biol. 132, 33-45 (2000).

50. Lander, G. C. et al. Appion: an integrated, database-driven pipeline to facilitate EM image processing. J. Struct. Biol. 166, 95-102 (2009).

51. Scheres, S. H. RELION: implementation of a Bayesian approach to cryo-EM structure determination. J. Struct. Biol. 180, 519-530 (2012).

52. Yu, G. et al. An algorithm for estimation and correction of anisotropic magnification distortion of cryo-EM images without need of pre-calibration. J. Struct. Biol. 195, 207-215 (2016).

53. Liu, Z., Guo, F., Wang, F., Li, T. C. \& Jiang, W. 2.9 Å resolution cryo-EM $3 \mathrm{D}$ reconstruction of close-packed virus particles. Structure 24, 319-328 (2016).
54. Sievers, F. et al. Fast, scalable generation of high-quality protein multiple sequence alignments using Clustal Omega. Mol. Syst. Biol. 7, 539 (2011).

55. Robert, X. \& Gouet, P. Deciphering key features in protein structures with the new ENDscript server. Nucleic Acids Res. 42, W320-W324 (2014)

\section{Acknowledgements}

We thank Yinguan Sun and Valorie D. Bowman (Purdue University) for technical help in cryoEM data collection, Yue Liu and Vidya Mangala Prasad (Purdue University) for advice in fitting of coordinates, and Chuan Xiao (University of Texas at El Paso) for assistance in drawing roadmaps. CryoEM data were collected at the Purdue University CryoEM facility. This work was supported by U.S. National Institutes of Health grant awards R01 AI076331 (to M.G.R.), R01 AI073755 (to M.S.D., sub-awards to R.J.K., M.G.R. and J.E.C.) and R01 AI104972 (to M.S.D.) as well as National Institutes of Health contract HHSN272201400024C (to J.E.C.).

\section{Author contributions}

S.S.H., A.M., G.S., T.K., F.L. and E.F. performed the experiments. S.S.H., T.K., J.C.P., A.F., E.F., M.S.D., J.E.C., R.J.K. and M.G.R. performed data analysis. W.J. made jspr available for reconstruction and refinement of cryoEM map. S.S.H., R.J.K. and M.G.R. wrote the initial draft of the manuscript with all authors contributing to editing into the final form.

\section{Additional information}

Supplementary Information accompanies this paper at http://www.nature.com/ naturecommunications

Competing financial interests: M.S.D. is a consultant for Inbios and Visterra, on the Scientific Advisory Boards of Moderna and OraGene, and a recipient of grants from Moderna and Visterra. J.E.C. is a consultant for Sanofi and Ridgeback Biotherapeutics, is on the Scientific Advisory Boards of PaxVax, CompuVax, GigaGen, Meissa Vaccines and is a recipient of research grants from Moderna and Sanofi. The remaining authors declare no competing financial interests.

Reprints and permission information is available online at http://npg.nature.com/ reprintsandpermissions/

How to cite this article: Hasan, S. S. et al. A human antibody against Zika virus crosslinks the E protein to prevent infection. Nat. Commun. 8, 14722 doi: 10.1038/ncomms14722 (2017).

Publisher's note: Springer Nature remains neutral with regard to jurisdictional claims in published maps and institutional affiliations.

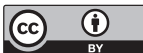

This work is licensed under a Creative Commons Attribution 4.0 International License. The images or other third party material in this article are included in the article's Creative Commons license, unless indicated otherwise in the credit line; if the material is not included under the Creative Commons license, users will need to obtain permission from the license holder to reproduce the material. To view a copy of this license, visit http://creativecommons.org/licenses/by/4.0/

(C) The Author(s) 2017 